\section{$\underset{\substack{\text { hommes } \\ \text { \& migrations }}}{ }$}

\section{Hommes \& migrations}

Revue française de référence sur les dynamiques

migratoires

1305 | 2014

L'exil chilien en France

\title{
L'accueil des exilés latino-américains en Europe
}

\section{Raúl Morales La Mura}

\section{(2) OpenEdition \\ Journals}

\section{Édition électronique}

URL : http://journals.openedition.org/hommesmigrations/2709

DOI : 10.4000/hommesmigrations.2709

ISSN : 2262-3353

\section{Éditeur}

Musée national de l'histoire de l'immigration

\section{Édition imprimée}

Date de publication : 1 janvier 2014

Pagination : $17-23$

ISBN : 978-2919-040261

ISSN : $1142-852 X$

\section{Référence électronique}

Raúl Morales La Mura, "L'accueil des exilés latino-américains en Europe », Hommes \& migrations [En ligne], 1305 | 2014, mis en ligne le 01 janvier 2017, consulté le 01 mai 2019. URL : http:// journals.openedition.org/hommesmigrations/2709; DOI : 10.4000/hommesmigrations.2709 


\title{
L'ACCUEIL DES EXILÉS LATINO-AMÉRICAINS EN EUROPE
}

par RAÚL MORALES LA MURA, sociologue et chercheur au Laboratoire lorrain de sciences sociales (2L2S), université de Lorraine.

\author{
Depuis un demi-siècle, les flux migratoires n'ont cessé \\ de se développer entre l'Amérique latine et l'Europe. \\ La révolution cubaine, l'avènement de la dictature chilienne et \\ le sort des exilés latino-américains ont ému les populations \\ européennes qui se sont montrées particulièrement \\ accueillantes. Mais, dans le contexte de crise économique \\ actuelle, les représentations changent et c'est à présent vers \\ I'Amérique latine que se tournent certains Européens en quête \\ d'une vie meilleure.
}

Depuis les pères de l'indépendance latino-américaine, l'Europe a toujours été une destination d'exil, dans toute l'acception du terme : exil que nous pouvons qualifier d'idéologique à la fin du XVIII siècle et au début du XIX'e, d'intellectuel au tout début du $\mathrm{XX}$ et de politique à la fin, puis d'économique dans la première décennie de ce XXI ${ }^{e}$ siècle. Mais cette relation n'est pas univoque et sa réciprocité est toute aussi prégnante, nous forçant à intégrer dans l'analyse l'existence durable, voire permanente, d'un flux migratoire pendulaire entre ces deux territoires socio-politiques et cela au gré de leurs vicis- situdes historiques. À l'exil des pères de l'indépendance, des intellectuels, des militants politiques et des anonymes à la recherche d'une viabilité économique, nous pouvons faire correspondre l'exil sur le territoire latino-américain de Guiseppe Garibaldi, ce "héros des deux mondes", et de ses compagnons ou encore celui des communards des deux Communes de Paris, des anarchistes italiens ou des républicains espagnols ${ }^{2}$. Sans oublier tous ceux qui chercheront une vie nouvelle dans le nouveau monde, séloignant raisonnablement des affres de la guerre et de ses néfastes conséquences écono- 
miques pour les populations ${ }^{3}$. La présente contribution ne s'intéressera quà une petite partie de cette longue histoire de migration alternée, celle de la deuxième moitié du XXe siècle et du tout début du XXIe. Sans la prétention de vouloir présenter de façon exhaustive les situations, les dispositifs ou les parcours, ni d'en faire un comparatif, nous mettrons en lumière quelques faits marquants ou emblématiques de ces deux périodes afin de montrer les dynamiques sociales qu'elles ont provoquées tant chez les accueillants que chez les arrivants.

En matière d'accueil des exilés latino-américains, deux événements ont marqué inégalement et indiscutablement l'Europe de la deuxième moitié du XXe siècle : la révolution cubaine et le coup d'État du 11 septembre 1973 au Chili. Si les effets du premier resteront limités géographiquement, leur intérêt n'est pas des moindres. Le second, au contraire, concernera l'ensemble de l'espace européen, alors que celui-ci était encore divisé entre l'Est et l'Ouest. L'émoi collectif suscité par cette migration forcée a obligé les politiques publiques à adapter leur offre en termes d'accueil, puis à repenser l'idéal type de l'exilé latino-américain.

\section{L'accueil des exilés politiques cubains en Espagne}

L'Espagne qui, pendant les quinze dernières années du régime franquiste, continue d'être un des pays d'émigration, donne asile à plus de 30000 Cubains ayant fui le tout nouveau régime castriste dès janvier 1959. Ce paradoxe peut être avancé comme l'une des raisons pour lesquelles la dictature espa- gnole n'a pu profiter de l'aura qu'elle pouvait espérer de cet effort pour accueillir les dissidents d'un régime communiste.

Tant à l'intérieur qu'à l'extérieur de la péninsule ibérique, une seule réalité sera retenue : les "restrictions" économiques et sociales, et la disparition des libertés politiques aboutissant à ce que nous pouvons considérer comme le quatrième exode espagnol du siècle ${ }^{5}$. Pour autant, la communauté cubaine en Espagne s'implantera sans encombre et prospérera sans se montrer politiquement. Elle tissera des liens avec la partie transatlantique de cette diaspora, sans jamais réussir à infléchir la politique étrangère de ce pays si accueillant qui, contre toute attente et au grand dam des États-Unis, maintiendra des liens diplomatiques qualifiés de "réguliers" avec Cuba.

\section{Quand I'Europe s'émeut}

Le reste de l'Europe des années 1970 suivait avec intérêt l'actualité chilienne jusqu'à ce moment inédit : la victoire par les urnes d'un Président au programme marxiste, puis le respect des institutions, dès son investiture et tout au long de son mandat. Sile contexte de bipolarisation produit par la guerre froide donnait comme improbable l'expérience démocratique de l'Unité populaire de Salvador Allende, celle-ci faisait tout de même écho aux aspirations de la gauche européenne à la recherche d'une expression propre face aux deux blocs. Ce fut le cas de la Suède socio-démocrate d'Olaf Palme, de l'Italie d'Enrico Berlinguer et d'Aldo Moro dans sa recherche d'un compromis historique entre communistes et démocrates-chrétiens, et également celui de la France avec une Union de la gauche qui présentait François Mitterrand comme candidat à l'élection présidentielle de 1974. L'expérience chilienne, même si elle était déclarée marxiste, 
était alors perçue par la gauche européenne comme une brèche possible dans les logiques hégémoniques issues de l'affrontement Est/Ouest. Le coup d'État, puis la dictature du général Pinochet stopperont avec brutalité et barbarie cette illusion. C'est là l'un des arguments invoqués pour tenter de comprendre l'émotion collective provoquée par cet événement en Europe, compte tenu de l'importance des demandes d'asile et des sentiments humanitaires ou d'injustice suscités par les images ${ }^{6}$ de la violence systématisée des putschistes à l'encontre des idées et des hommes qui les avaient exprimées. Des reportages sur le coup d'État lui-même et sur la répression qui a suivi ont été diffusés et utilisés par les militaires chiliens comme un instrument supplémentaire de répression par la terreur qu'ils pouvaient provoquer chez leurs opposants. Mais, inversement, les images du bombardement du palais présidentiel, des chars qui pointent leurs canons et leurs mitrailleuses sur des civils désarmés ou encore celles des militaires brûlant des livres ont réveillé l'inconscient collectif européen et rouvert les cicatrices de l'histoire contemporaine. Les raisons de cet émoi collectif et durable, dépassant le cadre même des affinités politiques, sont à chercher dans une analyse de toutes les données sans oublier le caractère propre des exilés eux-mêmes? 


\section{Les dynamiques sociales induites en Europe par l'accueil des exilés politiques chiliens}

Aucun pays européen, de l'Est ou de l'Ouest, ne répond par la négative à la demande d'asile des Chiliens. Chacun, avec ses réalités sociales, politiques et administratives particulières, reconnait le caractère abject de leur situation. Ces exilés seront quelque 20000 en Suède, 10000 en France, 7000 répartis de manière équitable entre les deux Allemagnes, 5000 en Grande-Bretagne, 3000 en
Italie, 2000 en Suisse, 1000 en Belgique, en Roumanie ou en Bulgarie, des centaines en Yougoslavie et moins d'une centaine au Luxembourg. Le compte $^{8}$ n'est ici qu'approximatif mais, au-delà de ces chiffres, il faut noter l'émergence, dans la société civile, de phénomènes allant de la désobéissance civique à l'influence politique, cela dans le but d'accueillir ces exilés sans considérer les limites imposées par les dispositifs ad hoc en place.

Nous pouvons rappeler l'effort de l'ambassadeur de Suède au Chili Harald Edelstam qui, à cause de ses positions et de son action, vit son ambassade attaquée militairement à plusieurs reprises, entre septembre et décembre 1973. La dynamique sociale est semblable quand nous considérons les comi- 
tés de solidarité formés en Europe par les nationaux. Citons le cas de la Suisse qui, après avoir fixé un nombre de Chiliens à accueillir, acceptera, à la demande de la société civile, d'en accueillir dix fois plus $^{9}$. On peut citer aussi le cas de la France, non parce que ses gouvernants ou son administration ont limité a priori le nombre de personnes à accueillir, mais parce que le champ associatif a œuvré à la mise en place d'un dispositif national d'accueil des exilés. Les exemples de ce type sont aussi variés que multiples sur l'ensemble du territoire européen. Cependant, ils présentent tous deux caractéristiques communes : ils sont le résultat d'une émotion collective et d'une action durable, pour le moins solidaire quand elle ne fut pas engagée, d'une partie conséquente de la société civile accueillante. Ce sont ces dynamiques sociales qui participeront à la reconfiguration de l'idéal type de l'exilé latino-américain.

\section{La particularité de l'Espagne dans l'accueil des exilés politiques}

Le dernier quart des années 1970 voit aussi l'arrivée en Europe d'exilés politiques d'autres pays latino-américains, fuyant des dictatures semblables idéologiquement à celle de Pinochet, avec les mêmes dispositions pour la brutalité et pour la violence instituée. Ces derniers arrivants bénéficieront des dispositifs d'accueil désormais conçus "pour" et expérimentés par la diaspora chilienne. Identifiés à l'idéal type de l'exilé, leurs conditions de vie et de socialisation seront semblables dans presque toute l'Europe, à l'exception de l'Espagne. L'Espagne post-franquiste se présente comme le choix majoritaire des Argentins, des Uruguayens, des Boliviens, des Colombiens ou des Péruviens, même si ce choix implique une plus forte précarité institutionnelle étant donné qu'il ne leur est

pas accordé le statut de réfugié. Les ressortissants argentins sont, par exemple, plus de 45000 en Espagne quand ils ne dépasseront jamais les 2500 en France ou en Suède ${ }^{10}$. Si les dynamiques sociales, qui ont émergé dans la société civile des autres pays européens pour accueillir les exilés politiques chiliens, se retrouvent dans la péninsule Ibérique, cette fois pour faire face à l'exil des Latino-Américains (Chiliens y compris), elles ne réussiront à produire leurs effets que bien trop tard. Ce n'est qu'en 1984 qu'un statut est accordé à ces exilés, avec la promulgation de la loi régulant le droit d'asile et la condition de réfugié. Entre-temps ils auront résidé avec un visa touristique renouvelé tous les trois

Ces exilés seront quelque 20000 en Suède, 10000 en France 7000 répartis de manière équitable entre les deux Allemagnes, 5000 en Grande-Bretagne, 3000 en Italie, 2000 en Suisse, 1000 en Belgique, en Roumanie ou en Bulgarie, des centaines en Yougoslavie et moins d'une centaine au Luxembourg. mois, sans véritables entraves administratives et tout en ayant le droit de travailler, suivant une législation datant de 1969 conçue pour faciliter la présence cubaine anticastriste.

\section{L'exil économique latino-américain en Europe, soudain et massif}

En lien avec l'actualité latino-américaine, la dernière décennie du XXe siècle voit apparaître une nouvelle problématique : celle du retour des exilés politiques $^{11}$. Puis, au début du XXI ${ }^{e}$ siècle, commence véritablement l'exil économique des Latino-Américains en Europe. Ce phénomène est à la fois soudain et massif, mais touche inégalement le territoire européen. Les pays émetteurs sont les pays andins ${ }^{12}$, essentiellement l'Équateur dès l'année 2000, et les pays d'accueil l'Espagne et l'Italie. L'Espagne 
accueillant presque un demi-million d'Équatoriens en 2006 et dix fois moins pour l'Italie. Pour le reste de l'Europe, même en considérant ici ou là que les effectifs des résidents des pays andins aient pu doubler voire tripler, ils n'arriveront jamais à l'ampleur notée pour ces deux pays.

Pour comprendre cette différence, il faut prendre en compte les causes mêmes de migrations, lesquelles peuvent être qualifiées d'ordinaires. D'une part, une crise économique en Équateur provoquée par la dollarisation de sa monnaie en 1999, a pour conséquence une dévaluation qui fait perdre plus de $30 \%$ de pouvoir d'achat aux Équatoriens. D'autre part, de ce côté-ci de l'Atlantique, principalement en Espagne et en Italie, un besoin de main-d'œuvre pour répondre à la demande des nouveaux marchés agricoles et de services d'aide à la personne. Il s'agit d'une migration fortement féminisée, jeune et qualifiée ${ }^{13}$. Ces femmes seules ou avec des enfants se concentrent dans les grandes agglomérations urbaines, Madrid, Barcelone, Rome, Milan et Gène, là où les besoins d'aide à la personne se font le plus sentir. Elles viennent compléter ou concurrencer la main-d'œuvre déjà existante ${ }^{14}$. On notera aussi leur présence en Andalousie, exclusivement pour les besoins de l'agriculture.

\section{La confrontation aux discriminations}

Dans les deux pays d'accueil privilégiés de cette migration, l'Espagne et l'Italie, les dynamiques sociales affrontées par les exilés sont proches des discriminations subies par les migrations polo-

Les nouveaux Latinos - Graffiti art Valparaiso (c) D.R.

naises, italiennes ou maghrébines en France. Elles finiront par réifier les Latinos au travers de qualificatifs comme "travailleuses" et "gentilles" pour les femmes, "machistes" et "alcooliques" pour les hommes, "délinquants" et "dangereux" pour les jeunes ${ }^{15}$. Cette perception négative va s'étendre à l'ensemble des Latino-Américains résidant dans ces deux pays ${ }^{16}$. Pour les sociétés d'accueil, il s'agit moins de se défendre que d'affirmer la domination des implantés sur les transplantés. Ces mécanismes sont une constante qui dépasse largement le cadre 
de cette contribution, ils ont été maintes fois étudiés à propos d'autres réalités géographiques, historiques et politiques ${ }^{17}$.

\section{La fin de l'exil économique latino-américain en Europe}

La crise financière de la fin de la première décennie du XXI siècle a touché particulièrement la Grèce, l'Italie et l'Espagne. Dans le même temps, l'Équateur connaît une embellie économique et institue une politique incitative au retour, portée par le Président lui-même. Il en est de même, dans une moindre mesure, pour les autres pays andins. Ainsi, entre 2008 et 2012, presque 12000 exilés des pays andins, établis en Espagne, sont retournés dans leur pays d'origine. À ce nombre, il faut ajouter les personnes qui ont choisi de passer d'une stratégie bipolaire à une autre, tripolaire, en allant chercher la stabilité économique Dans d'autres pays et notamment aux États-Unis, où la communauté andine est implantée de longue date. Rien qu'en 2012, plus de 150000 Équatoriens résidant en Espagne ont choisi de quitter le pays et on ne les retrouve pas parmi les effectifs recensés dans d'autres pays européens. Si ces facteurs marquent la fin de cet exil, les discriminations envers les Latino-Américains dans les pays d'accueil se poursuivent encore. Dans un marché du travail tellement restreint, les Européens commencent à leur tour à penser, peutêtre à décider, leur propre exil économique sur des territoires émergents comme le Chili, le Brésil ou l'Argentine. La France a accueilli essentiellement des exilés politiques et notamment chiliens. Si nous prenons pour référence le nombre d'exilés ayant bénéficié du statut de réfugié, en termes quantitatifs, elle se place en deuxième position derrière la Suède. De façon plus qualitative, les dynamiques sociales induites par cet accueil sont les mêmes sur le territoire français que dans le reste de l'Europe, mais se traduisent en France par un dispositif national d'accueil des exilés, organisé par l'administration de l'État et délégué en gestion à la société civile par l'intermédiaire de son champ associatif. C'est là un des facteurs qui a participé à l'intégration de ces populations, leur permettant de se construire un itinéraire social de reclassement qui les a amenées, pour la plupart, à retrouver au moins leur niveau social d'origine dans une société française qui méritait alors le qualificatif d'accueillante ${ }^{18}$.

\section{Conclusion}

$\mathrm{Si}$, pendant la deuxième moitié du $\mathrm{XX}^{\mathrm{e}}$ siècle et le tout début du XXIe, un nombre certain d'exilés latino-américains ont été accueillis sur le territoire européen, il ne faut pas méconnaître le caractère pendulaire de ce flux migratoire.

Les aléas historiques, politiDans un marché du travail tellement restreint, ques et économiques sont les Européens commencent déterminants en matière de à leur tour à penser, peut-être migration, figurant et modelantles déplacements des perà décider, leur propre exil économique sur des territoires sonnes d'un côté et de l'autre émergents comme le Chili, de l'Atlantique. le Brésil ou l'Argentine.

Dans cette logique, les études sociologiques et démographiques s'intéressent aujourd'hui aux Européens qui cherchent des nouvelles perspectives sur le territoire latino-américain. Ce flux migratoire pendulaire entre l'Amérique latine et l'Europe, tour à tour terres d'émigration et d'immigration, n'a donc pas de conclusion possible. 